\title{
Embedding Digital Chronotherapy into Bioelectronic Medicines
}

John E. Fleming ${ }^{1 *}$, Vaclav Kremen ${ }^{2,3,4}$, Ro'ee Gilron ${ }^{5 *}$, Nicholas M. Gregg ${ }^{2}$, Derk-Jan Dijk ${ }^{6,7}$, Philip A. Starr $^{5}$, Gregory A. Worrell ${ }^{2,4^{*}}$, Simon Little ${ }^{8 *}$, Timothy J. Denison ${ }^{1, *^{*}}$

1. Medical Research Council Brain Network Dynamics Unit, Nuffield Department of Clinical Neurosciences, University of Oxford, Mansfield Road, Oxford OX1 3TH, UK.

2. Bioelectronics Neurophysiology and Engineering Laboratory, Department of Neurology, Mayo Clinic, Rochester, MN, United States.

3. Cognitive Systems and Neurosciences, Czech Institute of Informatics, Robotics and Cybernetics, Czech Technical University in Prague, Prague, Czechia.

4. Department of Physiology and Biomedical Engineering, Mayo Clinic, Rochester, MN, United States.

5. Department of Neurological Surgery, University of California San Francisco, San Francisco, CA, United States.

6. Surry Sleep Research Centre, University of Surrey, University of Surrey, Guildford.

7. UK Dementia Research Institute Care Research and Technology Centre, Imperial College London and the University of Surrey.

8. Department of Neurology, University of California San Francisco, San Francisco, CA, United States.

9. Institute of Biomedical Engineering, Department of Engineering Science, University of Oxford, Oxford, United Kingdom.

*. These authors contributed equally

Biological rhythms permeate all living organisms at a variety of timescales. These rhythms are fundamental to physiological homeostasis, and their disruption is thought to play a key role in the initiation, progression, and expression of disease. In the last two decades, neuromodulation has been established as an effective adjunct therapy for medically refractory neurological disorders. To date, however, due to the limited sensing and algorithm capabilities of neuromodulation devices, exploring the influence of biological rhythms on therapy efficacy has not been feasible. However, with the development of new bioelectronic devices capable of long-term data recording and adaptive stimulation parameter adjustments, clinical neuroscience researchers are now gaining unprecedented insight into patient physiology across a variety of neurological diseases, including longitudinal rhythmic behavior. In this perspective, we propose that future bioelectronic devices 
should integrate chronobiological considerations in their physiological control structure to maximize the benefits of therapy. We specifically highlight this need for deep brain stimulation (DBS) chronotherapy, where the DBS therapeutic dosage would be titrated based on the time-ofday and synchronized to each patient's individual chronotype/sleep-wake cycle. This is motivated by preliminary longitudinal data recorded from both patients with Parkinson's disease (PD) and epilepsy, which show periodic symptom biomarkers synchronized to sub-daily (ultradian), daily (circadian $^{1}$ ), and longer time scale (infradian) rhythms. In addition, considering side effects, tonic stimulation can undermine diurnal patterns and cause fragmentation of sleep-wake rhythms. Based on these observations, we suggest a control structure for future bioelectronic devices which incorporates anticipatory, time-based adaptation of stimulation control, locked to patient-specific biological rhythms, as an adjunct to classical feedforward and feedback control methods. Initial results from three case studies using chronotherapy-enabled prototypes will illustrate the concept. The proposed control architecture for a future bioelectronic implant mimics more closely the classical integration of adaptive, feedforward, and feedback control methods found in physiology, and could be useful as a general method for personalized therapy refinement.

\section{Introduction}

Throughout physiology, homeostasis is observed at multiple spatial and temporal scales. Physiological processes such as body temperature, blood pressure and blood hormone concentrations are carefully regulated during healthy conditions, while dysregulation can lead to the initiation, progression, and expression of diseases (Smolensky and Peppas, 2007; Videnovic et

\footnotetext{
${ }^{1}$ Traditionally in biological rhythm research, rhythms with a near 24-hour period that are assessed and persist in the absence of external 24-hour rhythms are referred to as circadian rhythms. When 24-hour rhythms are observed in the presence of external 24-hour cycles, these rhythms are referred to as diurnal rhythms because strictly speaking it has not been demonstrated that these rhythms are generated endogenously.
} 
al., 2014; Leng et al., 2019). From a control system perspective, physiological homeostasis is maintained by a combination of feedforward, feedback, and adaptive control strategies (Houk, 1988; Crago et al., 1996; Wright et al., 2016), Figure 1. In a physiological control system, a feedback controller generates a forcing function that converges the system performance to a desired setpoint by continuously comparing the controlled variable, such as blood pressure or glucose level, to a target setpoint. Feedback controllers thus provide continuous negative feedback to counteract disturbances which would deviate the controlled variable from its target setpoint (Houk, 1988; Crago et al., 1996; Wright et al., 2016). The target setpoint is dictated by a feedforward controller which does not continuously monitor the system output. Rather, the feedforward controller operates in open-loop where its outputs are sent as command signals to the feedback controller or directly to the controlled system as generating functions. Thus, inputs to the feedforward controller specify the overall control objectives and target setpoints for the control process, such as blood pressure regulation (Houk, 1988; Tan and Taylor, 2011). Additionally, the feedforward controller may provide anticipatory control adjustments in response to monitored system disturbances, such as increases in blood flow during standing or exercise (Kitaoka et al., 2011; Matsukawa et al., 2012), or insulin control prior to eating a meal (Power and Schulkin, 2008). Feedforward and feedback control strategies alone are sufficient for the regulation of timeinvariant systems however may provide suboptimal performance for time-varying systems. Inclusion of adaptive controllers are necessary for the regulation of time-varying physiological systems. The role of the adaptive controller is to modify elements of the feedforward and feedback controllers based on slow or intermittent feedback information to promote beneficial alterations in the controlled system (Houk, 1988; Crago et al., 1996; Wright et al., 2016). 


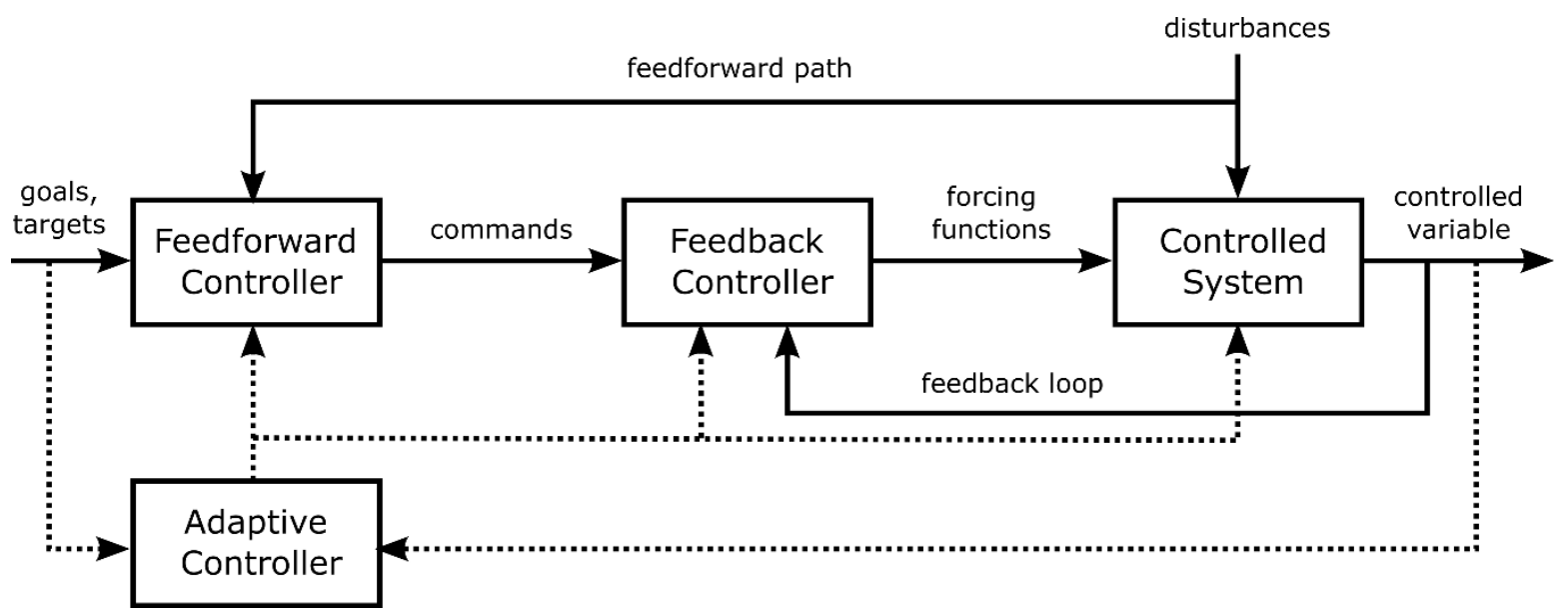

Figure 1: Control Structure for a physiological system. Homeostasis in physiological systems is maintained by a combination of adaptive, feedforward and feedback control strategies. This figure has been adapted from (Houk, 1988).

In contrast to the definition of adaptive control above, the term 'adaptive' is used throughout bioelectronic medicine to describe neuromodulation devices capable of instantaneous parameter adjustments in response to monitored physiomarker signals. These 'adaptive' devices in truth rely solely on static implementations of either feedforward, such as activity-based cardiac pacemakers (Dulk et al., 1988; Lloyd et al., 2017) and motion-adaptive spinal cord stimulation devices (Wagner et al., 2018), or feedback control strategies, such as responsive epilepsy stimulation (Sun and Morrell, 2014; Geller et al., 2017) and closed-loop DBS devices (Little et al., 2016a; Velisar et al., 2019). These devices do not adjust elements in their control structure to account for longer temporal variations encountered in physiology that may influence therapy outcomes. With improved sensing capabilities and access to longitudinal data in new neuromodulation devices it is becoming increasingly clear that in order to optimally regulate disease pathophysiology there is a necessity to realign our definition of 'adaptive' devices with those of physiological control systems.

\section{Biological Rhythms and Neuromodulation}

Although living organisms maintain constant homeostasis, their underlying physiological processes are not time invariant. Physiological processes display rhythmic variations which recur 
periodically at ultradian (less than 24 hours), circadian (24 hours), and infradian (> 24 hours, e.g. 7 day [circaseptan] rhythm time scales). and infradian (1 month) time scales. Circadian rhythms are generated endogenously with a spontaneous period close to but not exactly 24 hours. They are synchronized to zeitgebers, or cues from the external environment, the most notable of which is the light-dark cycle (Duffy and Wright Jr, 2005). The light-dark, via the retino-hypothalamic tract, synchronizes the master clock, located in the suprachiasmatic nucleus of the ventral hypothalamus, to a 24-hour period (Cederroth et al., 2019; Hastings et al., 2019; Kim et al., 2019). This master clock subsequently entrains circadian rhythms in a wide variety of behavioral and physiological variables, such as the sleep-wake cycle, core body temperature rhythm, the cortisol rhythm, cortical excitability, etc. In the absence of the light-dark cycle, and other zeitgebers, the master clock fails to entrain to a 24-hour period and results in free-running circadian rhythms, where the endogenous period of the circadian rhythm is greater than 24 hours. When entrained to a 24-hour period, variations in the timing of the sleep-wake and other circadian rhythms are also observed between individuals. These variations are referred to as an individual's chronotype, where a chronotype is defined as an individual's natural inclination to sleep at a particular phase of the 24hour day-night cycle (Roenneberg, 2012). Disruption of this sleep-wake circadian rhythm, and more specifically sleep architecture, has been associated with a range of disease pathophysiology (Steele et al., 2021). However, other rhythmic variations are also reported throughout disease pathophysiology, such as endogenous circadian fluctuations in symptom severity (Smolensky et al., 2015) and exogenous ultradian variations induced due to medication scheduling (Smolensky et al., 2021). Due to this, scheduling medication intake based on the time-of-day is an important consideration when optimizing patient-specific therapeutic outcomes, where medication dosages are scheduled to maximize therapeutic benefits and avoid disruption of the circadian sleep-wake cycle (Cardinali and Pandi-Perumal, 2006; Smolensky and Peppas, 2007; Videnovic et al., 2014; Ruben et al., 2019; Zaki et al., 2019; Smolensky et al., 2021).

Due to limited sensing capabilities of previous generations of bioelectronic devices the influence of biological rhythms on neuromodulation efficacy has been largely unexplored. Traditionally, therapy optimization for bioelectronic devices has been limited to daytime scheduled clinical visits, a single phase of the day-night cycle. As a result, tonic stimulation 
parameters, or control goals for 'adaptive' bioelectronic devices, may provide effective therapeutic benefits for the specific phase or the day-night cycle that they are optimized for, but may perform better or worse, and in worst cases induce side-effects, during other phases of the circadian cycle (Amara et al., 2012; Voges et al., 2015; Sharma et al., 2018). To illustrate this, in the following sections we highlight the presence of these biological rhythms in preliminary longitudinal data recorded from patients with PD and epilepsy during DBS therapy.

\section{Case Study 1 - Parkinson's Disease}

PD is one of the fastest growing neurological conditions with enormous human and economic costs. It is estimated that PD will affect 1.5 million people in the US and cost upwards of $\$ 79$ billion by 2037 (Yang et al., 2020). Medications are standard therapy for patients, where medication is generally provided in tablet form on a fixed schedule to replace or boost endogenous dopamine (Armstrong and Okun, 2020). However, despite optimal medical care, the majority of patients will experience fluctuations in their motor symptoms over time (Schrag and Quinn, 2000). For these patients advanced treatment options include dopaminergic infusions or DBS, which can reduce but usually do not eliminate fluctuations (Worth, 2013).

Over time, the management of PD becomes significantly orientated around the optimization of therapy towards symptom fluctuations that occur according to biological and exogenously driven rhythms. Although PD symptoms are often described to be improved on waking - the "sleep benefit" (Merello et al., 1997) - overall sleep quality and the sleep-wake circadian cycle in PD is notably disrupted (Mantovani et al., 2018). This is characterized by frequent awakenings and decreased time in both deep (N3) sleep and rapid eye movement stages (REM) as measured by extracranial and intracranial recordings of neural rhythms (Zahed et al., 2021). Treatment for PD is currently optimized for daytime motor control with both medications and DBS titrated to the awake state. These treatments therefore also impose intervention related ultradian rhythms on patients with PD. Dopaminergic medication is generally taken during the day on a fixed schedule between 2 and 4 hourly, which results in transitioning from OFF $\rightarrow$ ON $\rightarrow$ OFF states throughout the medication cycle. Before bed, patients often take a single long-acting 
dopaminergic medication to treat overnight akinesia, imposing a slow medication cycle overnight, although this has not been found to impact sleep microstructure (Wailke et al., 2011).

Overall, it has been found that open-loop DBS improves sleep structure as a fortuitous byproduct (Hjort et al., 2004). However, it is not currently optimized for nighttime or within sleep stages (Zahed et al., 2021). Recently, investigational trials have used newer sensing-enabled DBS devices to deliver closed-loop DBS in response to biomarkers correlated with motor performance (Little et al., 2016b; Velisar et al., 2019; Gilron et al., 2021). These closed-loop approaches are currently being orientated around daytime motor fluctuations but may lead to suboptimal therapy and sleep disruption at nighttime if the biomarker used to adjust stimulation is naturally reduced relative to its daytime counterpart, Figure 2. These circadian and rhythmic symptom fluctuations in PD could be addressed using a physiological control structure as detailed in Figure 1. An adaptive controller synchronized to patient-specific biological rhythms that are slower and relatively predictable in PD, such as the sleep-wake circadian cycle and ultradian medication cycles, could adjust elements of feedforward and feedback controllers to improve patient therapeutic outcomes for each phase of the 24-hour day-night cycle. In this manner, the feedforward and feedback controllers in the net control structure could accommodate nonrhythmic, unpredictable and/or fast disturbances encountered by DBS in a time-localized manner, where homeostasis of specific control goals or stimulation parameters is gradually varied throughout the sleep-wake cycle. 

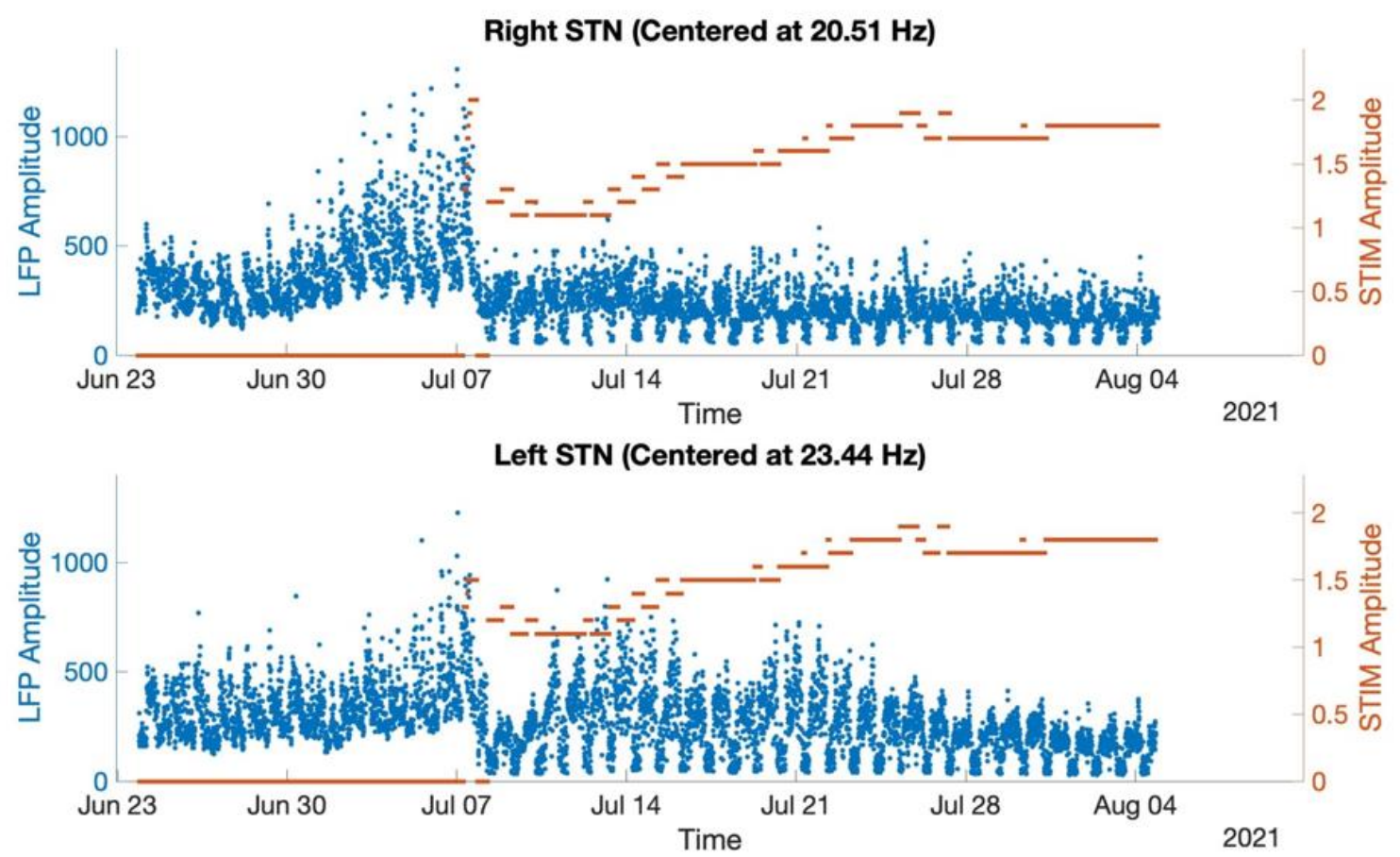

Figure 2: Chronic subcortical recordings from the subthalamic nucleus in a patient with Parkinson's disease. Bilateral subcortical recordings from a PD patient implanted with a sensing enabled DBS device, the Medtronic Percept PC ${ }^{\mathrm{TM}}$, tracking subcortical beta band power. Note the strong rhythmic circadian fluctuations in beta amplitude over the 24-hour cycle, in addition to the influence of stimulation intervention which suppresses beta and compresses the circadian beta fluctuation cycle.

\section{Case Study 2 - Epilepsy}

Epilepsy is a neurological disease in which brain activity becomes abnormal, resulting in seizures or periods of unusual behavior, sensations, and sometimes loss of awareness. Epilepsy affects close to 50 million people worldwide and has been estimated to cost the US economy $\$ 9.6$ billion annually (Yoon et al., 2009). Antiepileptic drugs are standard medical therapy for patients, however around one-third of patients are refractory to medication and continue to have sporadic seizures. For drug resistant patients, surgical interventions such as resective surgery or DBS are alternative options for seizure management. In practice, however resective surgery is not suitable for many drug resistant patients due to the epileptogenic brain region generating seizures being poorly localized, originating from multiple foci, or involving brain regions that cannot be safely 
resected. Furthermore, resective surgery is irreversible and only achieves seizure freedom in approximately $50 \%$ of operated patients overall (Téllez-Zenteno et al., 2005; Jehi et al., 2015). DBS is therefore an enticing option for these patients due to its reversible nature, where electrodes can be explanted with minimal side-effects if required. Duty cycle stimulation of the anterior nucleus of thalamus (ANT) and responsive neural stimulation, where stimulation is triggered by detected focal epileptiform activity, have received FDA approval for epilepsy (Fisher et al., 2010; Morrell, 2011; Bergey et al., 2015; Salanova et al., 2015).

The optimization of stimulation is however difficult and long-term seizure freedom is rare (Bergey et al., 2015; Nair et al., 2020; Salanova et al., 2021). The relevance of circadian rhythmicity in epilepsy has been recognised (Khan et al., 2018) but due to technology limitations of neuromodulation devices to-date, few clinical studies have investigated the rhythmicity and periodicity of seizures and their associated epileptiform brain activity. Optimization of patientspecific stimulation parameters has therefore been primarily driven by treatment failures which are estimated from seizure diaries collected by patients and their caregivers. The use of these diaries for monitoring seizure outcomes has been shown to demonstrate great inaccuracy, which in combination with the infrequent nature of seizures, has required physicians to optimize therapy over extended periods of time (Hoppe et al., 2007; Bergey et al., 2015; Blachut et al., 2015, 2017; Salanova et al., 2015). Access to newer sensing-enabled devices capable of chronic data recording are however highlighting a clearer role of biological rhythms in epilepsy (Baud et al., 2018; Gregg et al., 2020, 2021; Karoly et al., 2021). More specifically, long-term intracranial electroencephalogram (iEEG) recordings from human and canine patients demonstrate electrophysiological biomarkers with circadian and infradian seizure periodicities that occur independent of medication dosing that are believed to reflect endogenous rhythms associated with seizure risk (Baud et al., 2018; Karoly et al., 2018; Gregg et al., 2020), Figure 3.

Disruption of the sleep-wake cycle is also common in epilepsy, however stimulation at present is not optimized for nighttime or within sleep stage operation (Voges et al., 2015; Ruoff et al., 2020; Moore et al., 2021). ANT stimulation has been reported to disrupt sleep in a voltagedependent manner, where the frequency of arousals during sleep is positively correlated with the DBS voltage (Voges et al., 2015). Nighttime modulation of the DBS amplitude has thus been 
suggested as an approach to mitigate sleep architecture disruption during ANT stimulation. Similarly, patients with vagal nerve stimulators have been reported to demonstrate increased incidences of sleep apnea (Parhizgar et al., 2011). Due to this, vagal nerve stimulator devices such as the SenTiva from LivaNova have incorporated time-of-day based stimulation parameter adjustments to overcome this side-effect. Future stimulation devices for epilepsy may further improve therapeutic outcomes if stimulation parameters, and control goals, are dynamically adapted in this manner to reinforce sleep and other healthy brain rhythms. These devices may improve regulation of physiological brain activity to minimize seizure frequency for patients.

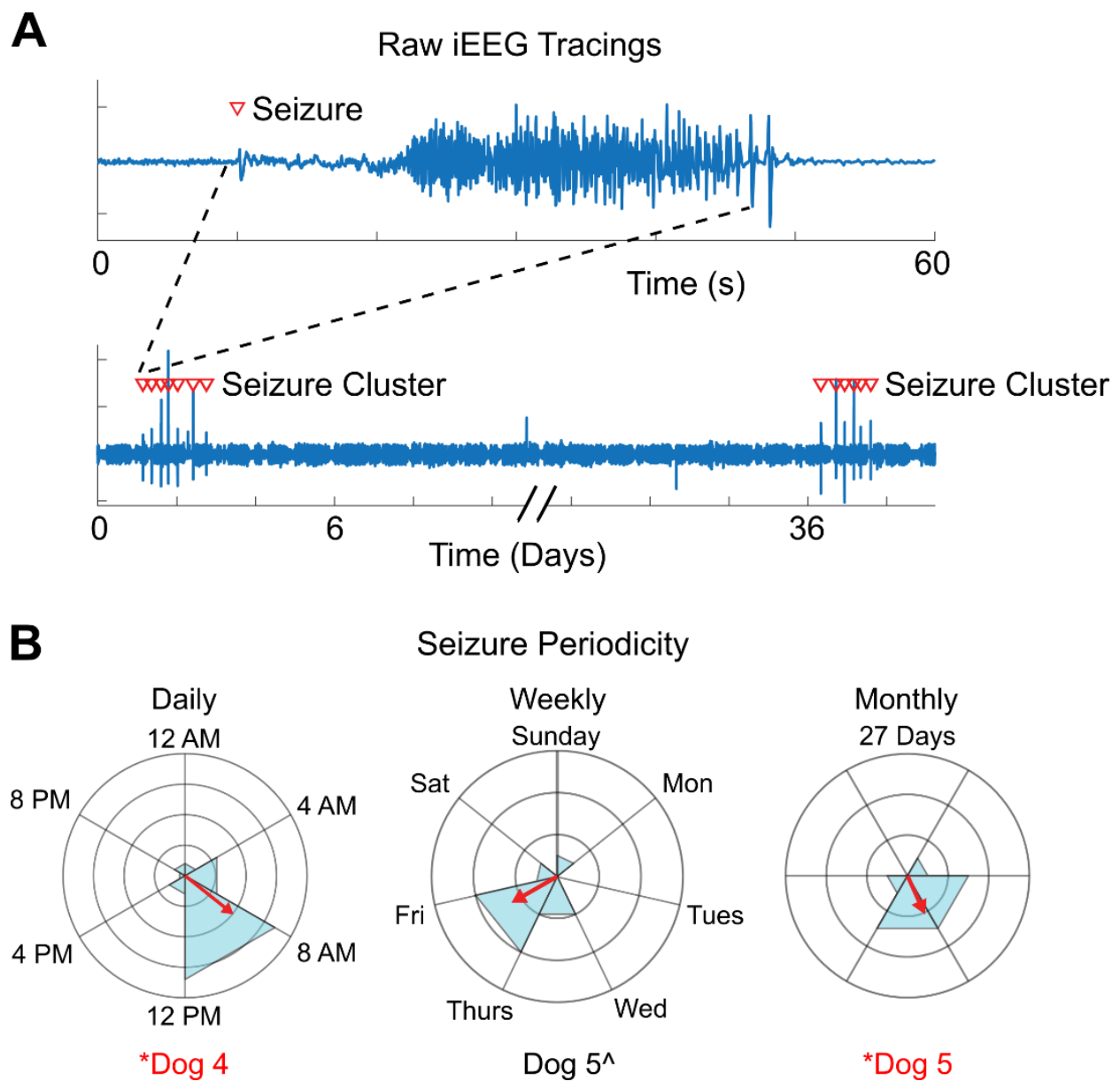

Figure 3: Example seizure periodicity in canine epilepsy. (A) Raw iEEG tracings. iEEG tracings are displayed at multiple time scales to illustrate a single seizure and a pair of seizure clusters separated by several days. Red triangles indicate seizure onset. (B) Circadian, circaseptan and monthly seizure periodicity. Daily, weekly, and monthly circular histograms of seizure occurrence. 
Concentric rings demarcate the number of seizures (five seizures per concentric ring in the daily histogram, two seizures per ring in the weekly and monthly histograms. The red bar is the resultant vector, or R-value. Dogs 4 and Dog 5 showed statistically significant daily and monthly periodicity, respectively, as indicated by the red font and asterisk. Dog 5 also indicates a trend towards significant weekly periodicity, however this did not survive statistical false discovery rate correction. This figure has been adapted from (Gregg et al., 2020).

\section{Future Adaptive Bioelectronic Devices}

The above case studies illustrate two examples of neurological disorders which demonstrate strong rhythmic variations across several timescales. Many other biological processes and neurological disorders are reported to display similar variations. For example, brain temperature, cortical excitability and brain responses to a performance task are all modulated by circadian and sleepwake cycles (Landolt et al., 1995; Ly et al., 2016; Muto et al., 2016), the circadian sleep-wake cycle modulates the threshold for pain perception (Hagenauer et al., 2017), while a variety of headache disorders are also synchronized to certain phases of the sleep-wake cycle (Burish et al., 2019; Naber et al., 2019). Patients with schizophrenia demonstrate significant disruption of circadian rhythms and sleep (Wulff et al., 2012), and there is considerable evidence of altered circadian rhythms, sleep disturbances and diurnal mood variation in patients with depression (Germain and Kupfer, 2008; Monteleone et al., 2011; Logan and McClung, 2018). Taken together, these studies suggest that future bioelectronic devices may improve patient therapeutic outcomes by integrating biological rhythm considerations into their control structures and realigning their implementations of adaptive control with the physiological control structure presented in Figure 1. More specifically, we propose that future neuromodulation devices should incorporate feedforward, feedback and adaptive controllers to regulate disease pathophysiology, where the implemented adaptive controller is synchronized to patient-specific rhythms, such as the patient's chronotype and medication schedule. In this manner the role of the adaptive controller is to adjust the device therapeutic control objectives based on the instantaneous phase of these rhythms, while the feedforward and feedback controllers regulate disease pathophysiology and intervention related side-effects in a time-localized manner by providing anticipatory and instantaneous adjustments to stimulation parameters. Overall integration of this physiological control structure 
in future devices would enable optimization of therapeutic outcomes over both short and long-time scales.

\section{Preliminary Implementations of Chronoadaptive Bioelectronic Devices}

Preliminary work has been undertaken to integrate patient-specific rhythms in DBS therapy. In the following sections we briefly highlight this work in two investigational DBS devices for the treatment of PD and epilepsy. The examples provided below highlight two technical approaches for the integration of chronoadaptation in DBS devices, namely fully embedded or distributed infrastructures.

\section{Investigational Medtronic Summit $\mathbf{R C}+\mathrm{S}^{\mathrm{TM}}$ - Sleep-Aware Adaptive DBS for PD}

Gilron et al. (2021) present an embedded sleep-aware adaptive DBS control system for PD implemented on an investigational Medtronic $\mathrm{RC}+\mathrm{S}$ implantable pulse generator (IPG) in (Gilron et al., 2021). In their study, four patients with PD were implanted bilaterally with cylindrical DBS leads in the subthalamic nucleus and paddle-type quadripolar leads in the subdural space over the motor cortex. The cortical and subcortical leads from each side were connected to an IPG which allowed independent control of each hemisphere. Two embedded classifiers on the IPG were programmed for the dual detection of neurophysiological biomarkers associated with sleep and the parkinsonian motor state, respectively, Figure 4 (A). The motor state classifier estimated "on" and “off" medication states for patients, where stimulation was reduced during on states to avoid stimulation induced dyskinesia and increased during off states to improve patient mobility (Gilron et al., 2021). When sleep was detected by the sleep classifier, the IPG delivered constant stimulation equivalent to clinically optimized open-loop stimulation regardless of the state of the motor state classifier. The interaction between the two classifiers was captured as a state table on the IPG, Figure 4 (B). The performance of the classifiers were verified using patient motor diaries, wearables and the $\mathrm{RC}+\mathrm{S}$ onboard accelerometer. In cases where the dual classifiers were deployed, the mean concordance between sleep measurements across both hemispheres was $88 \%$ (range 77 - $98 \%$ ). Additionally, the authors reported stable performance of the dual classifiers when tested 
over the course of 47 patient-days ( $24 \mathrm{~h}$ ) across four patients and six hemispheres. To assess the utility of a dedicated sleep classifier, the authors additionally simulated algorithm performance with omission of the sleep classifier. With its omission, all patients displayed large variation in the stimulation control signal during periods of sleep which could result in unwanted behavior depending on the algorithm used to control PD motor states during waking hours. 
A Dual motor \& sleep state detector - single day example
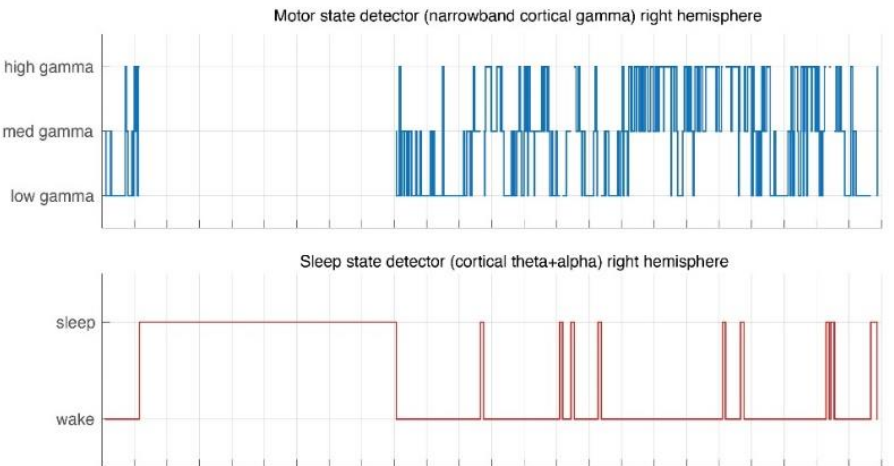

Current right hemisphere
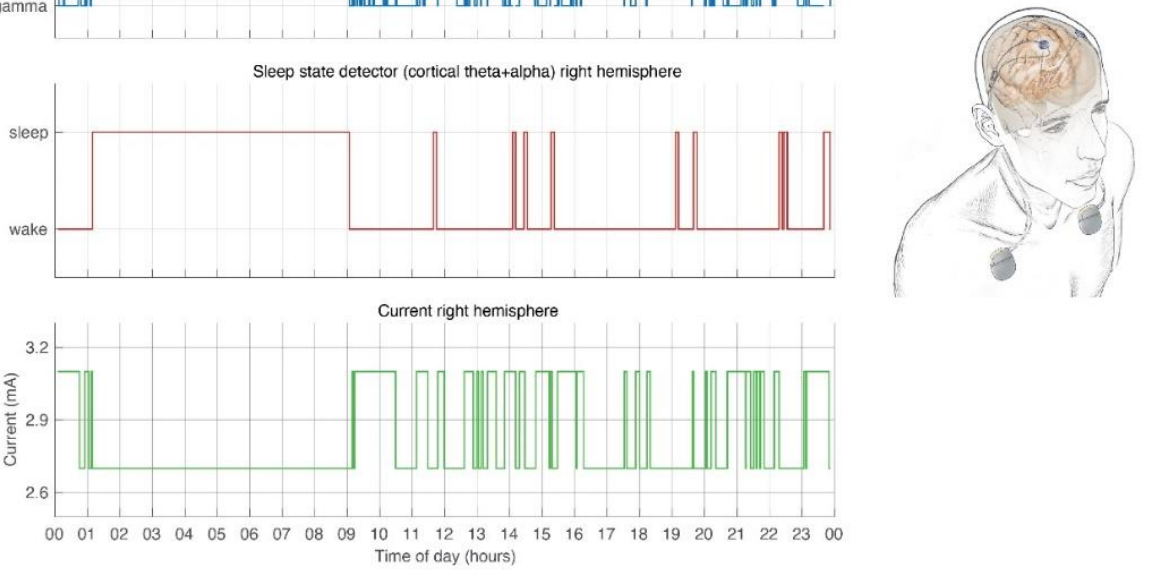

B

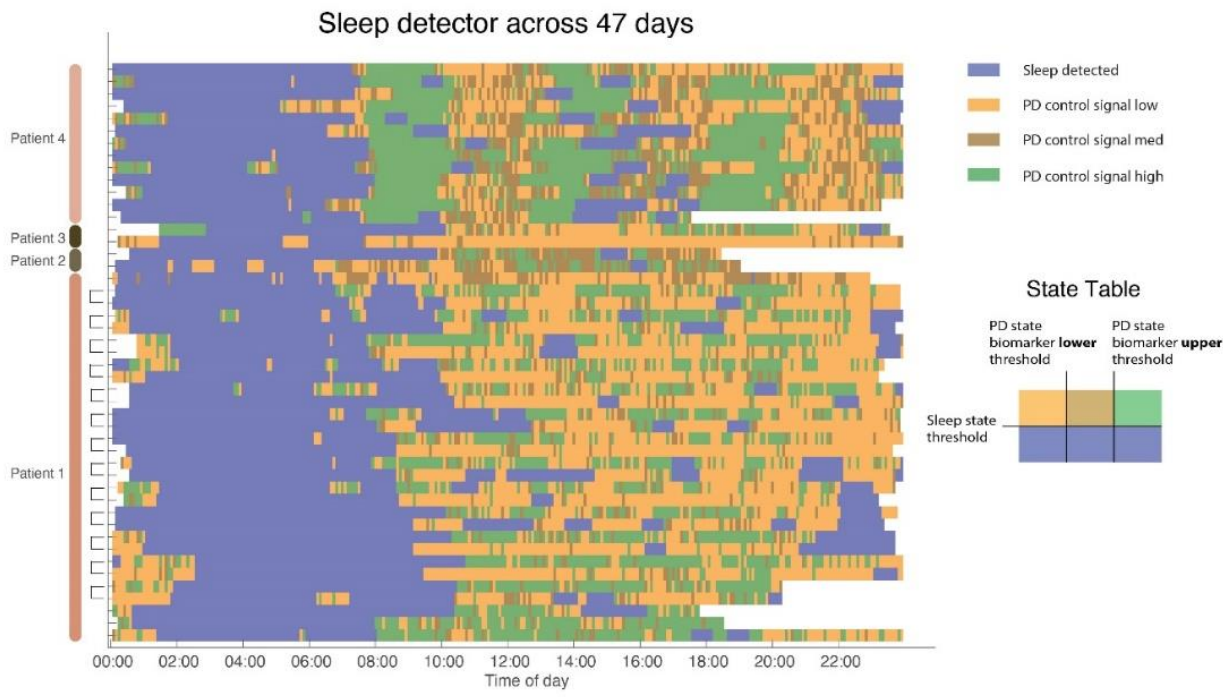

Figure 4: Fully-embedded sleep adaptive closed-loop DBS control in a patient with PD. (A) 24-hour performance of dual motor and sleep state classifiers for closed-loop amplitude modulation. When the sleep classifier detects sleep the motor state classifier is disabled and fixed amplitude open-loop stimulation is applied. Otherwise, when sleep is not detected the motor state classifier increases or reduces the stimulation amplitude when monitored cortical gamma activity is low or high, respectively. (B) Sleep detector performance over 47 days. The heatmap summarizes the sleep and motor state classifier performance over 47 days measured across four patients. Blue boxes indicate periods classified as sleep, while yellow and green boxes indicate the motor state as summarized in the state table. This figure is adapted from (Gilron et al., 2021) 


\section{Investigational Medtronic Summit $\mathrm{RC}+\mathrm{S}^{\mathrm{TM}}$ - Day/Night DBS Scheduling for Epilepsy}

Sleep co-morbidities are commonly experienced by patients with epilepsy. To improve sleep quality and seizure mitigation for patients with epilepsy the Mayo Epilepsy Personal Assistant Device (EPAD) is presented in (Attia et al., 2021; Sladky et al., 2021). The EPAD system is a distributed brain co-processor system that integrates implantable brain sensing and stimulation devices with off-the-body commercial electronics for clinical and neuroscience research applications (Attia et al., 2021; Sladky et al., 2021). The system overcomes computational and data storage limitations of bioelectronic devices with fully-embedded algorithms by providing wireless bi-directional connectivity between local tablet processors and distributed cloud computing technology (Kremen et al., 2018). The distributed system was investigated for the treatment of drug-resistant epilepsy in canine and human patients (Attia et al., 2021). The distributed system enabled continuous electrophysiology data streaming to a local tablet computer for real-time analysis and tracking of interictal epileptiform spike, seizures, and brain behavioral state coupled with patient reports to inform automatic adjustments of DBS parameters to optimize patient therapeutic outcomes (Kremen et al., 2018; Stanslaski et al., 2018; Attia et al., 2021; Mivalt et al., 2021). In prospective data recorded from one human and two canine ambulatory subjects, the interictal epileptiform spike detection algorithm, when compared to gold standard expert visually reviewed events, resulted in a sensitivity value of 0.9 and an F1-score of 0.81 (Sladky et al., 2021). The reported performance from the device seizure detector was also impressive with area under the curve precision recall values of $0.93,0.47$, and 0.88 for the human and two canine subjects, respectively (Sladky et al., 2021). The distributed system enabled the deployment of circadian adaptive control strategies where daytime stimulation parameters (amplitude and frequency) were adjusted to nighttime parameters optimized for minimizing sleep disruption (Attia et al., 2021; Sladky et al., 2021). 

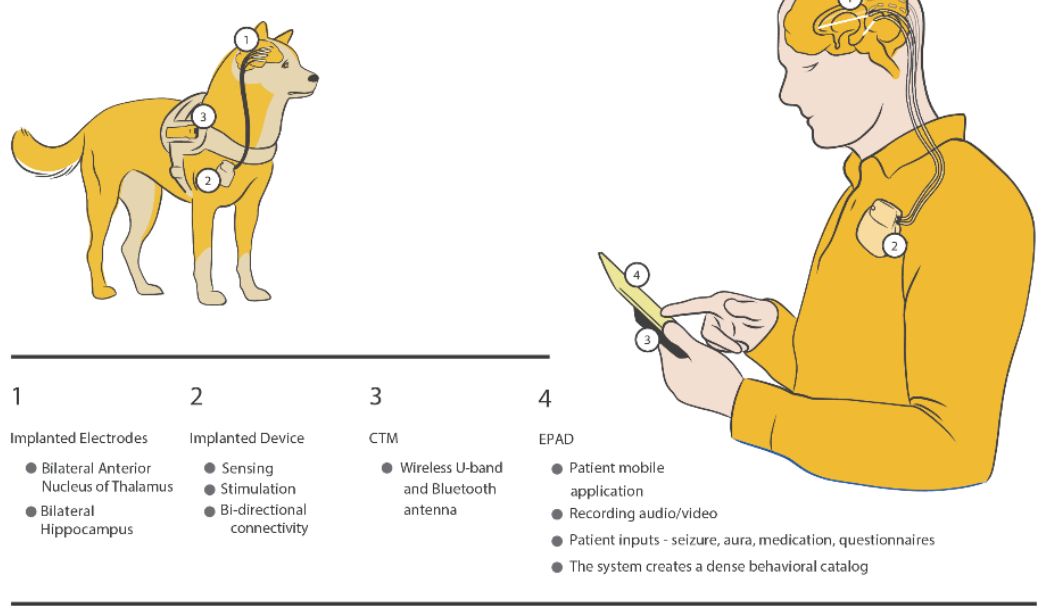

AUTOMATED SLEEP STAGING AND SLEEP-SPIKE CYCLES

SLEEP STAGE
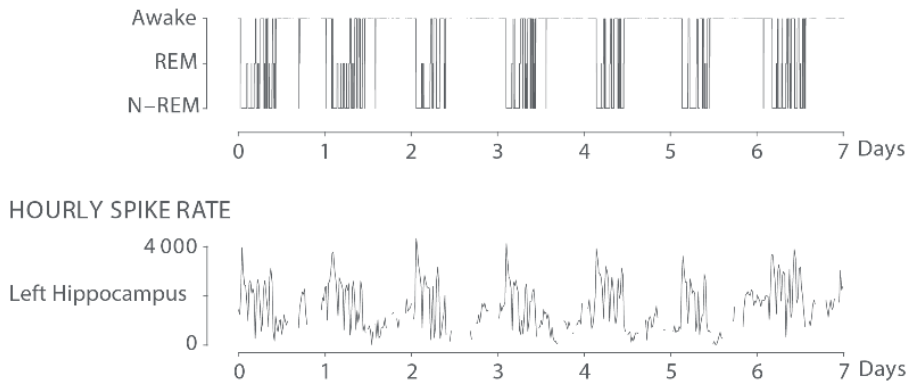

Figure 5: The Mayo EPAD distributed brain co-processor system for daytime/nighttime algorithm scheduling. The EPAD system provides bidirectional communication between implantable neuromodulation devices and commercially available electronics. The system has been investigated in both human and canine patients with epilepsy. The system is capable of sleep stage detection based on recorded neurophysiological data and enables targeted adaptation of stimulation during sleep.

\section{Picostim DyNeuMo-1 - Day/Night-DBS Control Scheduling for Epilepsy}

Zamora et al. (2021) investigated circadian adaptive DBS therapy in a case study of a canine with severe drug-resistant idiopathic generalized epilepsy that exhibited a characteristic nocturnal pattern correlated with its sleep-wake cycle (Zamora et al., 2021). Prior to DBS device implantation, the canine's cluster seizures evolved to status epilepticus and required emergency pharmacological intervention. The canine was implanted with a cranial-mounted DBS system (Toth et al., 2020; Zamora et al., 2020), whose electrodes were implanted bilaterally in the 
centromedian nucleus of the thalamus. The DBS system implanted in their case study was capable of both motion and time-based stimulation parameter adjustments fully-embedded on the device, as detailed in (Toth et al., 2020; Zamora et al., 2020) and illustrated below in Figure 6 (B). The DBS device was configured to deliver stimulation at specific frequencies characteristic of healthy neurophysiological activity and provide stimulation adjustments based on both the time-of-day and in response to activity. Post implantation, administration of the rescue medication levetiracetam was initially continued as a pulse therapy after seizure occurrence to prevent cluster seizure evolution or the occurrence of status epilepticus. In seven periods, stimulation, without rescue medication, was successful in disrupting cluster seizure emergence. The medication Phenobarbital was continued as chronic treatment over the whole observation time after implantation with a dose reduction from 13.3 to $12.5 \mathrm{mg} / \mathrm{kg} /$ day in November 2020 . At the time of writing their case study ( 7 months), the canine had experienced no further status epileptic events and no significant seizure clusters, Figure 6 (C). Further details regarding medication and stimulation parameters implemented during their case study can be found in the original publication (Zamora et al., 2021). 
A
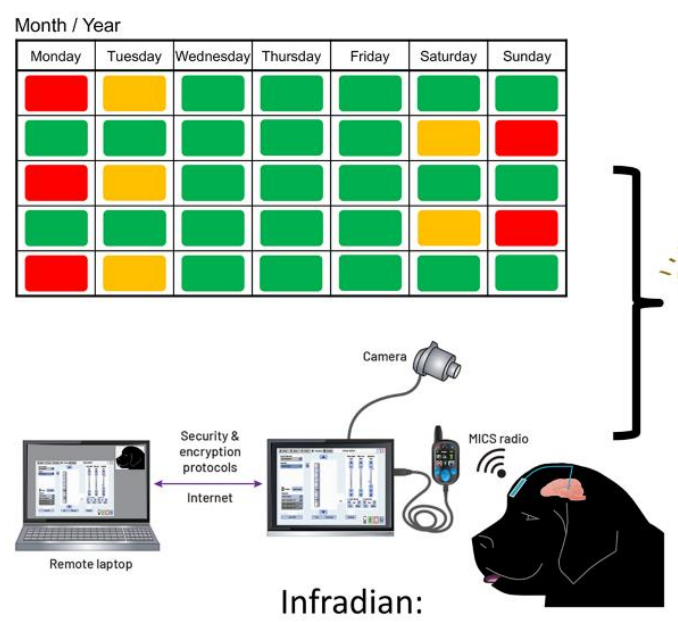

Distributed

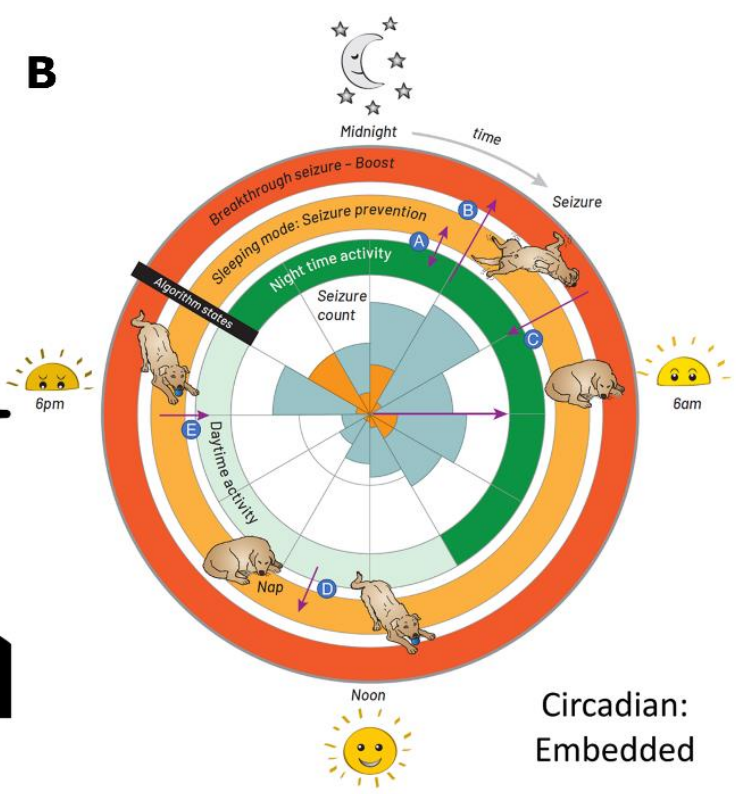

Circadian:

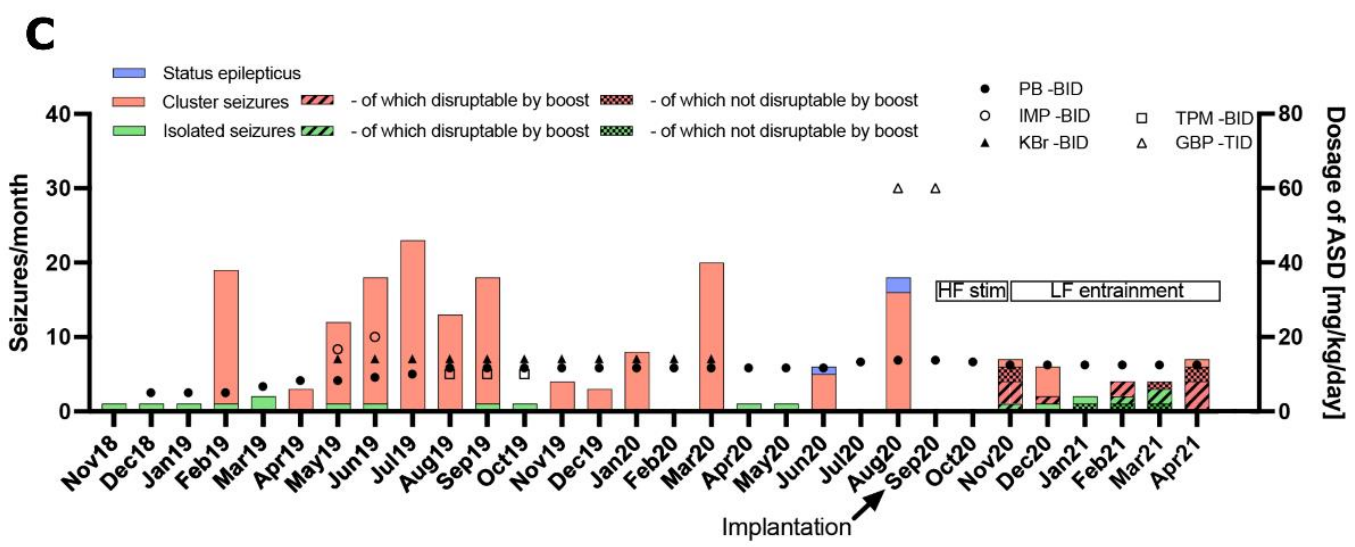

Figure 6: Example of DBS chronotherapy for canine epilepsy. (A) Infradian stimulation scheduling. The Picostim DyNeuMo1 system enables time-based scheduling of stimulation adjustments synchronized to the patient's particular infradian seizure rhythm (e.g. a 2-week period). (B) Rose plot illustration of embedded circadian stimulation algorithm adjustments. The inner circle represents the seizure count from the patient diary; the orange tiling is the timing of first seizure onset, while the blue account for all seizures in a cluster. The algorithm is composed of three states (represented as rings) to facilitate day/nighttime-based stimulation scheduling (inner green ring), a motion-triggered sleep mode to prevent seizure occurrence during daytime napping (middle pale orange ring), and a tap activated boost mode for preventing breakthrough seizures (outer dark orange ring). (C) Summary of canine seizure frequency and antiseizure drug dosage pre- and post-implantation. Post implantation the canine experience no status epilepticus events and no significant seizure clusters thus resulting in a reduction in rescue medication. Figures are adapted from (Zamora et al., 2021). 


\section{Future States and Limitations}

The above examples highlight two technology infrastructures for integrating chronotherapy in bioelectronic devices. In practice, however, future devices will require hybrid infrastructures to leverage the benefits of both embedded and distributed device functionalities. In this manner flexible device platforms will provide clinicians with improved insight into disease pathophysiology across a breadth of neurological disorders, whilst facilitating long-term optimization of neuromodulation chronotherapy on a disease- and patient-specific basis. Furthermore, flexible platforms such as this would enable the exploration of alternative, potentially computationally expensive algorithms to optimize stimulation parameters over time in accordance with the relevant biological rhythms, such as Bayesian optimization and machine learning techniques (Grado et al., 2018). The therapeutic performance of these algorithms may then be tracked over time and reviewed by clinicians at scheduled patient visits, while intermittent updates to algorithms could be wirelessly deployed over the cloud as necessary to provide seamless improvements in algorithm performance.

Limitations may however also be encountered in the development of these future chronotherapeutic bioelectronic devices. With the ability to trial new computationally expensive algorithms comes increased algorithm training complexity. Moreover, the ability to adapt the control strategy or stimulation parameters implemented in response to specific phases of biological rhythms may also result in increased clinical burden and programming time requirements. However, as highlighted in this article, the state-of-the-art for many neuromodulation therapies at present is still oriented around the delivery of open-loop stimulation optimized for maximizing daytime therapeutic benefits. Therefore, we propose that first generation chronoadaptive bioelectronic devices may readily improve patient therapeutic outcomes by simply implementing time-based adjustments to open-loop stimulation parameters aligned to patient-specific chronotypes and medication schedule. Following this, if further therapy refinement is necessary, additional feedforward- or feedback-based strategies can be layered in in a time-localized manner to provide additional stimulation refinement only when required. Deployment of chronoadaptation in this manner would thus immediately facilitate mitigation of suboptimal therapy performance at 
nighttime during sleep, whilst still maintaining daytime therapeutic benefits.

\section{Conclusion}

With the continued development of bioelectronic device technology, previous technical limitations that have limited access to longitudinal data recordings from patients with neurological disorders in the past are gradually being eased. As a result, clinical neuroscience researchers are beginning to gain insight into rhythmic variations in patient physiology over a variety of time scales and explore the influence of these biological rhythms on the therapeutic outcomes of neuromodulation therapies. With this in mind, it is becoming increasingly clear that there is a need to incorporate considerations for these biological rhythms in future bioelectronic devices. To optimize patientspecific therapeutic outcomes we emphasize that future devices should incorporate feedforward, feedback and adaptive control strategies, in combination, to maximize therapeutic benefits for patients and provide more natural regulation of patient pathophysiology to restore healthy physiological homeostasis.

\section{Author Contributions}

TD, SL and GW conceptualized the article concept. JF, VK and RG wrote the first draft of the manuscript. JF, VK, SL, RG, NG produced the article figures. All authors revised and approved the final version of the manuscript.

\section{Declaration of Interests}

JF, D-JD and PS declare no competing interests. VK consults for CertiCon. RG is an employee and shareholder of Rune Labs. NG and GW are investigators for the Medtronic Deep Brain Stimulation Therapy for Epilepsy Post-Approval. GW declares intellectual property licensed to Cadence Neuroscience. SL is a member of the scientific advisory board for RuneLabs and consults for Medtronic. The University of Oxford has research agreements with Bioinduction Ltd. TD also has business relationships with Bioinduction for research tool design and deployment. 


\section{References}

Amara, A. W., Standaert, D. G., Guthrie, S., Cutter, G., Watts, R. L., and Walker, H. C. (2012). Unilateral subthalamic nucleus deep brain stimulation improves sleep quality in Parkinson's disease. $\quad$ Parkinsonism \& $\quad$ Related $\quad$ Disorders $\quad 18, \quad 63-68$. doi:10.1016/J.PARKRELDIS.2011.09.001.

Armstrong, M. J., and Okun, M. S. (2020). Choosing a Parkinson Disease Treatment. JAMA 323, 1420-1420. doi:10.1001/JAMA.2020.1224.

Attia, T. P., Crepeau, D., Kremen, V., Nasseri, M., Guragain, H., Steele, S. W., et al. (2021). Epilepsy Personal Assistant Device-A Mobile Platform for Brain State, Dense Behavioral and Physiology Tracking and Controlling Adaptive Stimulation. Frontiers in Neurology 12. doi:10.3389/FNEUR.2021.704170.

Baud, M. O., Kleen, J. K., Mirro, E. A., Andrechak, J. C., King-Stephens, D., Chang, E. F., et al. (2018). Multi-day rhythms modulate seizure risk in epilepsy. Nature Communications 2017 9:1 9, 1-10. doi:10.1038/s41467-017-02577-y.

Bergey, G. K., Morrell, M. J., Mizrahi, E. M., Goldman, A., King-Stephens, D., Nair, D., et al. (2015). Long-term treatment with responsive brain stimulation in adults with refractory partial seizures. Neurology 84, 810-817. doi:10.1212/WNL.0000000000001280.

Blachut, B., Hoppe, C., Surges, R., Elger, C., and Helmstaedter, C. (2017). Subjective seizure counts by epilepsy clinical drug trial participants are not reliable. Epilepsy \& Behavior 67, 122-127. doi:10.1016/J.YEBEH.2016.10.036.

Blachut, B., Hoppe, C., Surges, R., Stahl, J., Elger, C. E., and Helmstaedter, C. (2015). Counting seizures: The primary outcome measure in epileptology from the patients' perspective. Seizure 29, 97-103. doi:10.1016/J.SEIZURE.2015.03.004.

Burish, M. J., Chen, Z., and Yoo, S.-H. (2019). Emerging relevance of circadian rhythms in headaches and neuropathic pain. Acta Physiologica 225, e13161. doi:10.1111/APHA.13161.

Cardinali, D. P., and Pandi-Perumal, S. R. (2006). Chronopharmacology and its implications to the pharmacology of sleep. Clinical pharmacology of sleep, 197-206. doi:10.1007/3-76437440-3_13. 
Cederroth, C. R., Albrecht, U., Bass, J., Brown, S. A., Dyhrfjeld-Johnsen, J., Gachon, F., et al. (2019). Medicine in the Fourth Dimension. Cell Metabolism 30, 238-250. doi:10.1016/J.CMET.2019.06.019.

Crago, P., Lan, N., Veltink, P., Abbas, J., and Kantor, C. (1996). New control strategies for neuroprosthetic systems. Journal of rehabilitation research and development 33, 158-172. Available at: https://www.academia.edu/download/44009834/crago.pdf [Accessed October $28,2021]$.

Duffy, J., and Wright Jr, K. (2005). Entrainment of the human circadian system by light. Journal of biological rhythms 20, 326-338. doi:10.1177/0748730405277983.

Dulk, K. den, Bouwels, L., Lindemans, F., Rankin, I., Brugada, P., and Wellens, H. J. J. (1988). The activitrax rate responsive pacemaker system. The American Journal of Cardiology 61, 107-112. doi:10.1016/0002-9149(88)91314-8.

Fisher, R., Salanova, V., Witt, T., Worth, R., Henry, T., Gross, R., et al. (2010). Electrical stimulation of the anterior nucleus of thalamus for treatment of refractory epilepsy. Epilepsia 51, 899-908. doi:10.1111/J.1528-1167.2010.02536.X.

Geller, E. B., Skarpaas, T. L., Gross, R. E., Goodman, R. R., Barkley, G. L., Bazil, C. W., et al. (2017). Brain-responsive neurostimulation in patients with medically intractable mesial temporal lobe epilepsy. Epilepsia 58, 994-1004. doi:10.1111/EPI.13740.

Germain, A., and Kupfer, D. J. (2008). Circadian rhythm disturbances in depression. Human Psychopharmacology: Clinical and Experimental 23, 571-585. doi:10.1002/HUP.964.

Gilron, R., Little, S., Wilt, R., Perrone, R., Anso, J., and Starr, P. A. (2021). Sleep-Aware Adaptive Deep Brain Stimulation Control: Chronic Use at Home With Dual Independent Linear Discriminate Detectors. Frontiers in Neuroscience 1307. doi:10.3389/FNINS.2021.732499.

Grado, L. L., Johnson, M. D., and Netoff, T. I. (2018). Bayesian adaptive dual control of deep brain stimulation in a computational model of Parkinson's disease. PLoS Computational Biology 14, 1-23. doi:10.1371/journal.pcbi.1006606.

Gregg, N. M., Nasseri, M., Kremen, V., Patterson, E. E., Sturges, B. K., Denison, T. J., et al. (2020). Circadian and multiday seizure periodicities, and seizure clusters in canine epilepsy. 
Brain Communications 2. doi:10.1093/BRAINCOMMS/FCAA008.

Gregg, N. M., Sladky, V., Nejedly, P., Mivalt, F., Kim, I., Balzekas, I., et al. (2021). Thalamic deep brain stimulation modulates circadian and infradian cycles of seizure risk in epilepsy. medRxiv, 2021.08.25.21262616. doi:10.1101/2021.08.25.21262616.

Hastings, M. H., Maywood, E. S., and Brancaccio, M. (2019). The Mammalian Circadian Timing System and the Suprachiasmatic Nucleus as Its Pacemaker. Biology 2019, Vol. 8, Page 13 , 13. doi:10.3390/BIOLOGY8010013.

Hjort, N., Østergaard, K., and Dupont, E. (2004). Improvement of sleep quality in patients with advanced Parkinson's disease treated with deep brain stimulation of the subthalamic nucleus. Movement Disorders 19, 196-199. doi:10.1002/MDS.10639.

Hoppe, C., Poepel, A., and Elger, C. E. (2007). Epilepsy: Accuracy of Patient Seizure Counts. Archives of Neurology 64, 1595-1599. doi:10.1001/ARCHNEUR.64.11.1595.

Houk, J. C. (1988). Control strategies in physiological systems. The FASEB Journal 2, 97-107. doi:10.1096/FASEBJ.2.2.3277888.

Jehi, L., Friedman, D., Carlson, C., Cascino, G., Dewar, S., Elger, C., et al. (2015). The evolution of epilepsy surgery between 1991 and 2011 in nine major epilepsy centers across the United States, Germany, and Australia. Epilepsia 56, 1526-1533. doi:10.1111/EPI.13116.

Karoly, P. J., Goldenholz, D. M., Freestone, D. R., Moss, R. E., Grayden, D. B., Theodore, W. H., et al. (2018). Circadian and circaseptan rhythms in human epilepsy: a retrospective cohort study. The Lancet Neurology 17, 977-985. doi:10.1016/S1474-4422(18)30274-6.

Karoly, P. J., Rao, V. R., Gregg, N. M., Worrell, G. A., Bernard, C., Cook, M. J., et al. (2021). Cycles in epilepsy. Nature Reviews Neurology 2021 17:5 17, 267-284. doi:10.1038/s41582021-00464-1.

Khan, S., Nobili, L., Khatami, R., Loddenkemper, T., Cajochen, C., Dijk, D. J., et al. (2018). Circadian rhythm and epilepsy. The Lancet Neurology 17, 1098-1108. doi:10.1016/S14744422(18)30335-1.

Kim, P., Oster, H., Lehnert, H., Schmid, S. M., Salamat, N., Barclay, J. L., et al. (2019). Coupling the Circadian Clock to Homeostasis: The Role of Period in Timing Physiology. Endocrine Reviews 40, 66-95. doi:10.1210/ER.2018-00049. 
Kitaoka, K., Miura, H., Kitamura, M., Akutagawa, M., Kinouchi, Y., and Yoshizaki, K. (2011). Feed-forward changes in carotid blood flow velocity during active standing. Neuroscience Letters 487, 240-245. doi:10.1016/J.NEULET.2010.10.030.

Kremen, V., Brinkmann, B. H., Kim, I., Guragain, H., Nasseri, M., Magee, A. L., et al. (2018). Integrating brain implants with local and distributed computing devices: A next generation epilepsy management system. IEEE Journal of Translational Engineering in Health and Medicine 6. doi:10.1109/JTEHM.2018.2869398.

Landolt, H., Moser, S., Wieser, H., Borbély, A., and Dijk, D. (1995). Intracranial temperature across 24-hour sleep-wake cycles in humans. Neuroreport 6, 913-917. doi:10.1097/00001756-199504190-00022.

Leng, Y., Musiek, E. S., Hu, K., Cappuccio, F. P., and Yaffe, K. (2019). Association between circadian rhythms and neurodegenerative diseases. The Lancet Neurology 18, 307-318. doi:10.1016/S1474-4422(18)30461-7.

Little, S., Beudel, M., Zrinzo, L., Foltynie, T., Limousin, P., Hariz, M., et al. (2016a). Bilateral adaptive deep brain stimulation is effective in Parkinson's disease. Journal of Neurology, Neurosurgery \& Psychiatry 87, 717-721. doi:10.1136/JNNP-2015-310972.

Little, S., Beudel, M., Zrinzo, L., Foltynie, T., Limousin, P., Hariz, M., et al. (2016b). Bilateral adaptive deep brain stimulation is effective in Parkinson's disease. Journal of Neurology, Neurosurgery and Psychiatry 87, 717-721. doi:10.1136/jnnp-2015-310972.

Lloyd, M., Reynolds, D., Sheldon, T., Stromberg, K., Hudnall, J. H., Demmer, W. M., et al. (2017). Rate adaptive pacing in an intracardiac pacemaker. Heart Rhythm 14, 200-205. doi:10.1016/J.HRTHM.2016.11.016.

Logan, R. W., and McClung, C. A. (2018). Rhythms of life: circadian disruption and brain disorders across the lifespan. Nature Reviews Neuroscience 2018 20:1 20, 49-65. doi:10.1038/s41583-018-0088-y.

Ly, J. Q. M., Gaggioni, G., Chellappa, S. L., Papachilleos, S., Brzozowski, A., Borsu, C., et al. (2016). Circadian regulation of human cortical excitability. Nature Communications 2016 7:1 7, 1-10. doi:10.1038/ncomms11828.

Mantovani, S., Smith, S. S., Gordon, R., and O’Sullivan, J. D. (2018). An overview of sleep and 
circadian dysfunction in Parkinson's disease. Journal of Sleep Research 27, e12673. doi:10.1111/JSR.12673.

Matsukawa, K., Liang, N., and Ishii, K. (2012). Central command: Feedforward control of the sympathoadrenal system during exercise. The Journal of Physical Fitness and Sports Medicine 1, 573-577. doi:10.7600/JPFSM.1.573.

Merello, M., Hughes, A., Colosimo, C., Hoffman, M., Starkstein, S., and Leiguarda, R. (1997). Sleep benefit in parkinson's disease. Movement Disorders 12, 506-508. doi:10.1002/MDS.870120405.

Mivalt, F., Kremen, V., Sladky, V., Balzekas, I., Nejedly, P., Gregg, N., et al. (2021). Electrical Brain Stimulation and Continuous Behavioral State Tracking in Ambulatory Humans. medRxiv, 2021.08.10.21261645. doi:10.1101/2021.08.10.21261645.

Monteleone, P., Martiadis, V., and Maj, M. (2011). Circadian rhythms and treatment implications in depression. Progress in Neuro-Psychopharmacology and Biological Psychiatry 35, 1569 1574. doi:10.1016/J.PNPBP.2010.07.028.

Moore, J. L., Carvalho, D. Z., St Louis, E. K., and Bazil, C. (2021). Sleep and Epilepsy: a Focused Review of Pathophysiology, Clinical Syndromes, Co-morbidities, and Therapy. Neurotherapeutics 2021 18:1 18, 170-180. doi:10.1007/S13311-021-01021-W.

Morrell, M. J. (2011). Responsive cortical stimulation for the treatment of medically intractable partial epilepsy. Neurology 77, 1295-1304. doi:10.1212/WNL.0B013E3182302056.

Muto, V., Jaspar, M., Meyer, C., Kussé, C., Chellappa, S. L., Degueldre, C., et al. (2016). Local modulation of human brain responses by circadian rhythmicity and sleep debt. Science 353, 687-690. doi:10.1126/SCIENCE.AAD2993.

Naber, W. C., Fronczek, R., Haan, J., Doesborg, P., Colwell, C. S., Ferrari, M. D., et al. (2019). The biological clock in cluster headache: A review and hypothesis. Cephalalgia 39, 18551866. doi:10.1177/0333102419851815.

Nair, D. R., Laxer, K. D., Weber, P. B., Murro, A. M., Park, Y. D., Barkley, G. L., et al. (2020). Nine-year prospective efficacy and safety of brain-responsive neurostimulation for focal epilepsy. Neurology 95, e1244-e1256. doi:10.1212/WNL.0000000000010154.

Parhizgar, F., Nugent, K., and Raj, R. (2011). Obstructive Sleep Apnea and Respiratory 
Complications Associated with Vagus Nerve Stimulators. Journal of Clinical Sleep Medicine 7, 401-407. doi:10.5664/JCSM.1204.

Power, M. L., and Schulkin, J. (2008). Anticipatory physiological regulation in feeding biology: Cephalic phase responses. Appetite 50, 194-206. doi:10.1016/J.APPET.2007.10.006.

Pritchard, C., Rosenorn-Lanng, E., Silk, A., and Hansen, L. (2017). Controlled population-based comparative study of USA and international adult [55-74] neurological deaths 1989-2014. Acta Neurologica Scandinavica 136, 698-707. doi:10.1111/ANE.12789.

Ray Dorsey, E., Elbaz, A., Nichols, E., Abd-Allah, F., Abdelalim, A., Adsuar, J. C., et al. (2018). Global, regional, and national burden of Parkinson's disease, 1990-2016: a systematic analysis for the Global Burden of Disease Study 2016. The Lancet Neurology 17, 939-953. doi:10.1016/S1474-4422(18)30295-3.

Roenneberg, T. (2012). What is chronotype? Sleep and biological rhythms 10, 75-76. Available at: https://www.academia.edu/download/46217552/j.1479-8425.2012.00541.x201606049608-o3o4p.pdf [Accessed August 26, 2021].

Ruben, M. D., Smith, D. F., FitzGerald, G. A., and Hogenesch, J. B. (2019). Dosing time matters. Science 365, 547-549. doi:10.1126/SCIENCE.AAX7621.

Ruoff, L., Jarosiewicz, B., Zak, R., Tcheng, T. K., Neylan, T. C., and Rao, V. R. (2020). Sleep disruption is not observed with brain-responsive neurostimulation for epilepsy. Epilepsia Open 5, 155-165. doi:10.1002/EPI4.12382.

Salanova, V., Sperling, M. R., Gross, R. E., Irwin, C. P., Vollhaber, J. A., Giftakis, J. E., et al. (2021). The SANTÉ study at 10 years of follow-up: Effectiveness, safety, and sudden unexpected death in epilepsy. Epilepsia 62, 1306-1317. doi:10.1111/EPI.16895.

Salanova, V., Witt, T., Worth, R., Henry, T. R., Gross, R. E., Nazzaro, J. M., et al. (2015). Longterm efficacy and safety of thalamic stimulation for drug-resistant partial epilepsy. Neurology 84, 1017-1025. doi:10.1212/WNL.0000000000001334.

Schrag, A., and Quinn, N. (2000). Dyskinesias and motor fluctuations in Parkinson's diseaseA community-based study. Brain 123, 2297-2305. doi:10.1093/BRAIN/123.11.2297.

Sharma, V. D., Sengupta, S., Chitnis, S., and Amara, A. W. (2018). Deep Brain Stimulation and Sleep-Wake Disturbances in Parkinson Disease: A Review. Frontiers in Neurology 0, 697. 
doi:10.3389/FNEUR.2018.00697.

Sladky, V., Nejedly, P., Mivalt, F., Brinkmann, B. H., Kim, I., Louis, E. K. st., et al. (2021). Distributed Brain Co-Processor for Neurophysiologic Tracking and Adaptive Stimulation: Application to Drug Resistant Epilepsy. bioRxiv, 2021.03.08.434476. doi:10.1101/2021.03.08.434476.

Smolensky, M. H., Hermida, R. C., and Geng, Y. J. (2021). Chronotherapy of cardiac and vascular disease: timing medications to circadian rhythms to optimize treatment effects and outcomes. Current Opinion in Pharmacology 57, 41-48. doi:10.1016/J.COPH.2020.10.014.

Smolensky, M. H., and Peppas, N. A. (2007). Chronobiology, drug delivery, and chronotherapeutics. Advanced Drug Delivery Reviews 59, 828-851. doi:10.1016/J.ADDR.2007.07.001.

Smolensky, M. H., Portaluppi, F., Manfredini, R., Hermida, R. C., Tiseo, R., Sackett-Lundeen, L. L., et al. (2015). Diurnal and twenty-four hour patterning of human diseases: acute and chronic common and uncommon medical conditions. Sleep Medicine Reviews 21, 12-22. doi:10.1016/J.SMRV.2014.06.005.

Stanslaski, S., Herron, J., Chouinard, T., Bourget, D., Isaacson, B., Kremen, V., et al. (2018). A Chronically Implantable Neural Coprocessor for Investigating the Treatment of Neurological Disorders. IEEE Transactions on Biomedical Circuits and Systems 12, 1230-1245. doi:10.1109/TBCAS.2018.2880148.

Steele, T. A., St Louis, E. K., Videnovic, A., and Auger, R. R. (2021). Circadian Rhythm SleepWake Disorders: a Contemporary Review of Neurobiology, Treatment, and Dysregulation in Neurodegenerative Disease. Neurotherapeutics 2021 18:1 18, 53-74. doi:10.1007/S13311021-01031-8.

Sun, F., and Morrell, M. (2014). The RNS System: responsive cortical stimulation for the treatment of refractory partial epilepsy. Expert review of medical devices 11, 563-572. doi:10.1586/17434440.2014.947274.

Tan, C. O., and Taylor, J. A. (2011). Feedback and feedforward sympathetic haemodynamic control: chicken or egg? The Journal of Physiology 589, 1867-1867. doi:10.1113/JPHYSIOL.2011.208199. 
Téllez-Zenteno, J. F., Dhar, R., and Wiebe, S. (2005). Long-term seizure outcomes following epilepsy surgery: a systematic review and meta-analysis. Brain 128, 1188-1198. doi:10.1093/BRAIN/AWH449.

Toth, R., Zamora, M., Ottaway, J., Gillbe, T., Martin, S., Benjaber, M., et al. (2020). DyNeuMo Mk-2: An Investigational Circadian-Locked Neuromodulator with Responsive Stimulation for Applied Chronobiology. in IEEE Transactions on Systems, Man, and Cybernetics: Systems (Institute of Electrical and Electronics Engineers Inc.), 3433-3440. doi:10.1109/SMC42975.2020.9283187.

Velisar, A., Syrkin-Nikolau, J., Blumenfeld, Z., Trager, M. H., Afzal, M. F., Prabhakar, V., et al. (2019). Dual threshold neural closed loop deep brain stimulation in Parkinson disease patients. Brain Stimulation. doi:10.1016/j.brs.2019.02.020.

Videnovic, A., Lazar, A. S., Barker, R. A., and Overeem, S. (2014). 'The clocks that time us'circadian rhythms in neurodegenerative disorders. Nature Reviews Neurology 2014 10:12 10, 683-693. doi:10.1038/nrneurol.2014.206.

Voges, B. R., Schmitt, F. C., Hamel, W., House, P. M., Kluge, C., Moll, C. K. E., et al. (2015). Deep brain stimulation of anterior nucleus thalami disrupts sleep in epilepsy patients. Epilepsia 56, e99-e103. doi:10.1111/EPI.13045.

Wagner, F. B., Mignardot, J.-B., le Goff-Mignardot, C. G., Demesmaeker, R., Komi, S., Capogrosso, M., et al. (2018). Targeted neurotechnology restores walking in humans with spinal cord injury. Nature 563, 65-71. doi:10.1038/s41586-018-0649-2.

Wailke, S., Herzog, J., Witt, K., Deuschl, G., and Volkmann, J. (2011). Effect of controlled-release levodopa on the microstructure of sleep in Parkinson's disease. European Journal of Neurology 18, 590-596. doi:10.1111/J.1468-1331.2010.03213.X.

Worth, P. F. (2013). When the going gets tough: how to select patients with Parkinson's disease for advanced therapies. Practical Neurology 13, 140-152. doi:10.1136/PRACTNEUROL2012-000463.

Wright, J., Macefield, V. G., van Schaik, A., and Tapson, J. C. (2016). A Review of Control Strategies in Closed-Loop Neuroprosthetic Systems. Frontiers in Neuroscience 0, 312. doi:10.3389/FNINS.2016.00312. 
Wulff, K., Dijk, D.-J., Middleton, B., Foster, R. G., and Joyce, E. M. (2012). Sleep and circadian rhythm disruption in schizophrenia. The British Journal of Psychiatry 200, 308-316. doi:10.1192/BJP.BP.111.096321.

Yang, W., Hamilton, J. L., Kopil, C., Beck, J. C., Tanner, C. M., Albin, R. L., et al. (2020). Current and projected future economic burden of Parkinson's disease in the US. npj Parkinson's Disease 6, 1-9.

Yoon, D., Frick, K. D., Carr, D. A., and Austin, J. K. (2009). Economic impact of epilepsy in the United States. Epilepsia 50, 2186-2191. doi:10.1111/J.1528-1167.2009.02159.X.

Zahed, H., Zuzuarregui, J. R. P., Gilron, R., Denison, T., Starr, P. A., and Little, S. (2021). The Neurophysiology of Sleep in Parkinson's Disease. Movement Disorders 36, 1526-1542. doi:10.1002/MDS.28562.

Zaki, N. F. W., Yousif, M., Bahammam, A. S., Spence, D. W., Bharti, V. K., Subramanian, P., et al. (2019). Chronotherapeutics: Recognizing the Importance of Timing Factors in the Treatment of Disease and Sleep Disorders. Clinical Neuropharmacology 42, 80-87. doi:10.1097/WNF.0000000000000341.

Zamora, M., Meller, S., Kajin, F., Sermon, J. J., Toth, R., Benjaber, M., et al. (2021). Case Report: Embedding "Digital Chronotherapy" Into Medical Devices-A Canine Validation for Controlling Status Epilepticus Through Multi-Scale Rhythmic Brain Stimulation. Frontiers in Neuroscience 0, 1196. doi:10.3389/FNINS.2021.734265.

Zamora, M., Toth, R., Ottaway, J., Gillbe, T., Martin, S., Benjaber, M., et al. (2020). DyNeuMo Mk-1: A Fully-Implantable, Motion-Adaptive Neurostimulator with Configurable Response Algorithms. bioRxiv, 2020.09.10.292284. doi:10.1101/2020.09.10.292284. 